\title{
Towards a Greater Understanding of Epilepsy-The Human Epilepsy Project
}

\author{
An Expert Interview with Ruben I Kuzniecky \\ Zucker Hofstra Northwell Health School of Medicine, New York, NY, USA
}

DOI: https://doi.org/10.17925/USN.2019.15.2.60

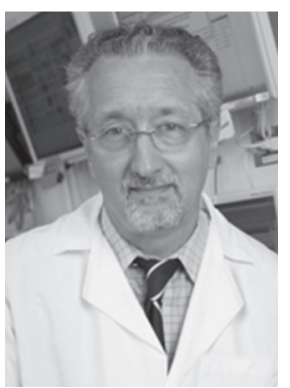

\section{Ruben I Kuzniecky}

Ruben I Kuzniecky is Professor and Vice Chair, Academic Affairs, and Director, Clinical Research Program, Department of Neurology, Zucker Hofstra Northwell Health School of Medicine, New York. He was the co-director and director of epilepsy research at the NYU Comprehensive Epilepsy Center between 2013-2017. He has authored over 300 books, chapters, and journal articles on a number of topics related to epilepsy, and has received epilepsy research grants from the National Institutes of Health and numerous foundations. His research focuses on brain imaging, malformations of brain development and epilepsy, and biomarkers of epilepsy. Prof. Kuzniecky has been recognized for his efforts in the "Best Doctors in America" multiple times and with many honorary lectures around the world. He is also the founder of LUCES, a foundation that provides epilepsy medications at no cost to poor families in Panama.

\section{Keywords}

Epilepsy, Human Epilepsy Project, biomarkers, focal seizures

Disclosure: Ruben I Kuzniecky is a lead investigator in the Human Epilepsy Project.

Review Process: This is an expert interview and, as such, has not undergone the journal's standard peer review process.

Acknowledgment: Medical writing assistance was provided by Katrina Mountfort of Touch Medical Media, and supported by Touch Medical Media.

Authorship: The named author meets the International Committee of Medical Journal Editors (ICMJE) criteria for authorship of this manuscript, takes responsibility for the integrity of the work as a whole, and has given final approval to the version to be published.

Received: September 5, 2019

Published Online: October 4, 2019

Citation: US Neurology, 2019;15(2):60-1

Corresponding Author: Ruben I Kuzniecky,

Zucker Hofstra School of Medicine, Northwell Health, Lenox Hill Hospital, 130 East 77 St, New York, NY 10075, USA. E.rkuzniecky@northwell.edu

Facebook: @HumanEpilepsyProject

Support: No funding was received in

the publication of this article. pilepsy is an important cause of disability and mortality, and affects around 50 million people worldwide. ${ }^{1}$ Focal seizures, which originate in only one part of the brain, account for around $80 \%$ of all epileptic seizures. ${ }^{2}$ Treating epilepsy, however, is challenging since the types, severity frequency, and impact of unprovoked seizures vary from person to person. One-third of people with epilepsy live with uncontrollable seizures because no available treatment works for them. ${ }^{3}$ Furthermore, we currently have no biomarkers to inform treatment decisions.

The Epilepsy Study Consortium and the Epilepsy Foundation are undertaking the Human Epilepsy Project (HEP), a study of focal seizures, to better understand the challenges of living with focal seizures and to determine biomarkers of treatment response.,5 In an expert interview, Prof. Kuzniecky discusses the aims and design of the HEP.

\section{Q. What do you consider the biggest challenges in the management of epilepsy?}

The challenges are accurate diagnosis and, more importantly, the correct etiology of epilepsy, which we are still unable to fully recognize, despite the availability of new imaging and molecular tools As a result, we can't tell around half our patients why they developed epilepsy. In addition, we still treat epilepsy the way we did 30 years ago, using broad spectrum drugs, and a trial-and-error mentality, not knowing in advance what the best treatment options are for that particular patient. We therefore use an empirical treatment strategy that depends on the patient's response in terms of outcome and side effects.

\section{Q. Could you tell us a little about the aims and design of the Human Epilepsy Project?}

The study started with the aim of trying to identify biomarkers in patients who develop focal epilepsy without an obvious risk factor, such as a tumor or stroke. These patients are commonly seen in clinical practice and have no major risk factors but develop epilepsy at some point in their life. We wanted to concentrate on patients with no clear etiology. We are interested in biomarkers for outcome and progression early in the disease process. HEP has created a large repository of patient information as well as imaging, biomarkers, DNA, and other assets that are likely to be useful in the future, to try to elucidate factors that can inform treatment decisions early in the disease and predict outcomes. 
A total of 490 patients have been enrolled in the HEP. One of the problems with many of the published studies on epilepsy is that they involve a mixed group of patients, e.g., patients with no clear evidence of epilepsy by laboratory measures, but a clinical diagnosis. In the HEP study, each patient without clear etiology or an abnormal electroencephalogram was required to be identified, classified and reviewed by a committee. Patients were asked to monitor seizures and medications via smartphone app, so we could continually track this information. Physicians were free to choose medications for each patient.

\section{Q. What are the inclusion and exclusion criteria for this study?}

Participants had to have confirmed focal epilepsy, and either no previous treatment, or treatment that had just been initiated $(<4$ months before enrollment). We included younger patients (age range 12-60 years) but not children and not older adults. We excluded patients with obvious reasons such as tumors, head trauma, and strokes. We also excluded patients with primary generalized epilepsy, and epilepsy with mental retardation/intellectual disability, as these could confound the analysis.

\section{Q. What have been the major findings of the HEP study, to date?}

HEP 1 was initiated in 2012 and enrollment ended in September 2018, so at the moment we have limited data on patients who have completed more than 2 years; we are not yet ready to release data. However, we have learned that only the minority of patients have a clear etiology; it is evident in less than $20 \%$ of magnetic resonance imaging scans. Additionally, in approximately one-third of patients, the brain appeared to be a little atrophic compared with normal controls. The structural brain changes perhaps suggest that the brain has undergone accelerated aging. ${ }^{6}$

Few of our patients are good trackers, meaning we have to spend a lot of time encouraging them to use the app for monitoring. If a patient became seizure-free or almost seizure-free, their motivation to track was of course lower.

In terms of treatment, levetiracetam is one of the most common anti-epileptic drugs used in the world for a number of reasons. It has a good safety and tolerability profile and most patients are likely to remain on it. HEP data, however, suggest that some patients are sensitive to levetiracetam while others are not, but the same is not true for other anticonvulsant drugs. The percentage of patients who had to switch from lamotrigine, oxcarbazepine or phenytoin to another drug was lower than those taking levetiracetam, when this drug was the first treatment.

The full data set from HEP 1 will be reported when all data from year 1 have been analyzed; this is expected in the coming year. Following this, we anticipate the 3-year and 5-year reports. We also plan to look at anti-inflammatory biomarkers from serum and genomic studies.

\section{Q. What does the HEP 2 study involve?}

Unlike HEP 1, HEP 2 is a retrospective study but patients are followed for 2 years to determine changes in clinical status e.g. intractability to non-intractability, though this does not seem to occur often. The HEP 2 study has been ongoing for around 1 year and is currently open for

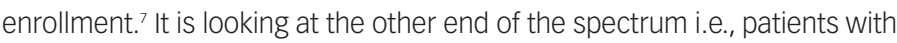
intractable epilepsy, and aims to retrospectively determine biomarkers that may tell us why they do not respond to treatment. We have strict criteria for intractability, that is, having failed four or more drugs at an acceptable therapeutic range, a very high seizure frequency, and at least two seizures a month i.e., severe. The study excludes patients with progressive diseases, brain tumors etc.

We anticipate being able to use HEP 1 and 2 to help us solve some of the practical issues facing patients and physicians in patients with focal epilepsy. $\square$

1. GBD 2016 Epilepsy Collaborators. Global, regional, and national burden of epilepsy, 1990-2016: a systematic analysis for the Global Burden of Disease Study 2016. Lancet Neurol. 2019;18:357-75.

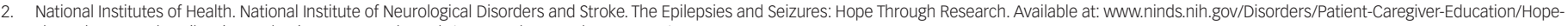
Through-Research/Epilepsies-and-Seizures-Hope-Through (accessed September 25, 2019).

3. Laxer KD, Trinka E, Hirsch $\sqcup$, et al. The consequences of refractory epilepsy and its treatment. Epilepsy Behav. 2014;37:59-70.

4. The Human Epilepsy Project. Available at: www.humanepilepsyproject.org/ (accessed September 25, 2019).

5. ClinicalTrials.gov. The Human Epilepsy Project (HEP). Available at: https://clinicaltrials.gov/ct2/show/NCT02126774 (accessed September 25, 2019).

6. Pardoe HR, Cole JH, Blackmon K, et al., Structural brain changes in medically refractory focal epilepsy resemble premature brain aging. Epilepsy Res. 2017;133:28-32

7. ClinicalTrials.gov. Human Epilepsy Project 2: Resistant Focal Seizures Study (HEP2). Available at: https://clinicaltrials.gov/ct2/show/NCT03531008 (accessed September 25, 2019). 